\title{
Diseño y validación de una escala para medir el estilo controlador del estudiante de Educación Superior
}

\author{
Elisa HUÉSCAR HERNÁNDEZ ${ }^{1}$, Juan Antonio MORENO-MURCIA ${ }^{1}$ \\ y Antonio ALÍAS GARCÍA ${ }^{2}$ \\ ${ }^{1}$ Universidad Miguel Hernández de Elche, ${ }^{2}$ Universidad de Almería.
}

(Recibido 21 Enero, 2017; Aceptado 18 Marzo, 2017)

\begin{abstract}
RESUMEN: El objetivo fue el diseño y validación de un instrumento de evaluación del estilo interpersonal controlador del estudiante de Educación Superior. Participaron 332 estudiantes universitarios (140 chicos y 192 chicas) de $2^{\circ}$ curso de seis grados pertenecientes al área de Ciencias de la Salud con una edad media de 21.05 años $(D T=3.97)$. Tras varias fases y el apoyo en análisis factoriales (exploratorios y confirmatorios) la Escala de Control del Estudiante ECE quedó conformada por un solo factor de ocho ítems. Los resultados indican que la escala presenta validez de constructo, fiabilidad y validez predictiva, ya que respecto a la variable sexo, las estrategias de interacción de control por parte de los chicos aparecen como más frecuentes que para las chicas. La escala ECE se presenta como válida y fiable, recomendando su uso en la valoración de los estilos de interacción de los estudiantes.
\end{abstract}

Palabras clave: Estudiante; Teoría Autodeterminación; Sexo.

Design and validation of a scale to assess the student's control style of higher education

\begin{abstract}
The goal was the design and validation of an instrument of interpersonal style evaluation of student driver Higher Education. Involved 332 college students (140 boys and 192 girls) 2nd year 6 degrees belonging to the area of Health Sciences with an average of 21.05 years (SD = 3.97). Afterseveralphases and support factor analysis (exploratory and confirmatory) (SCS) was composed of a single factor of eight items. The results indicate that the scale has construct validity, reliability and predictive validity, as compared to the variable sex, interaction control strategies by the boys appear more frequently than girls. The scale generated is presented as valid and reliable, recommending its use in assessing interaction styles of students.

Keywords: Student; Self-determination Theory; Sex.

${ }^{1}$ Correspondencia: Elisa Huéscar Hernández. Universidad Miguel Hernández de Elche. Departamento de Psicología de la Salud. Edificio Altamira. Avenida de la Universidad, s/n. 03202 Elche (Alicante). E-mail: ehuescar@umh.es. Tel: 966658525.
\end{abstract}




\section{Introducción}

Con el claro objetivo de situar al estudiante como el verdadero protagonista de su aprendizaje, las últimas leyes educativas (LOGSE, 1990, LOE, 2006, LOMCE, 2013), vienen planteando desde un enfoque constructivista la recomendación de dotar al discente de las competencias necesarias para que pueda construir y producir su propio conocimiento. Paralelamente, el papel del docente se sitúa en la actualidad en el foco de numerosas investigaciones que buscan acercarse al conocimiento de las estrategias más eficaces que satisfagan las necesidades de los estudiantes (Pegalajar y López, 2015). A partir de ambos planteamientos, el escenario de enseñanza-aprendizaje podría partir de la consideración de ambos promotores para comenzar a cimentar su desarrollo óptimo, pero, sin embargo, en ocasiones, el protagonismo en la literatura científica del rol docente soslaya el impulso de trabajos sobre las necesidades de los estudiantes respecto a sus intereses académicos, sociales o emocionales. En este sentido, algunos trabajos han señalado por ejemplo la relevancia de los vínculos entre estudiantes respecto a la repercusión sobre los aprendizajes (García, 2006) apoyándose en la importancia de naturaleza social de este constructo. Así pues, la interacción entre estudiantes la configuran una serie de patrones comportamentales individuales junto a los que se va a ir construyendo el conocimiento, siendo necesario profundizar en su comprensión para que el docente, desde una posición mediadora, atienda al fomento del reclamado carácter activo y autónomo del estudiante.

Estos patrones o estilos de interacción personal han sido conceptualizados desde el marco de la Teoría de la Autodeterminación TAD (Deci y Ryan, 1985, 1987) a partir de los dos extremos de un continuo, situando en uno de ellos al estilo de apoyo a la autonomía y en el otro extremo al estilo controlador. El estilo interpersonal de apoyo a la autonomía consiste en que la persona tiene en consideración los puntos de vista de los demás a la hora de gestionar una tarea, mostrándose empático con ella y ofreciendo diferentes posibilidades de elección a partir de las necesidades de los demás (Black y Deci, 2000; Deci y Ryan, 1985). Por su parte, el estilo controlador, utiliza presiones, amenazas para imponer sus pensamientos y sentimientos a los demás. Dichas estrategias de coerción pueden incluir recompensas implícitas, explícitas o castigos (Black y Deci, 2000). En la actualidad, en el contexto educativo (Hagger y Chatzisarantis, 2007; Niemiec, Ryan, y Deci, 2009) la mayoría de trabajos focalizan en el estilo de apoyo a la autonomía por las consecuencias positivas que genera como la facilitación de los afectos positivos (Bartholomew, Ntoumanis, y Thogersen-Ntounami, 2011), el autoconcepto (Coastsworth y Conray, 2009), el rendimiento académico (Boggiano, Flink, Shields, Seelbach, y Barrett, 1993) o el bienestar (Adie, Duda, y Ntoumanis, 2012). Sin embargo, el conocimiento sobre el estilo interpersonal controlador viene siendo más limitado (Bartholomew, Ntoumanis, Ryan, Bosch, y Thogerse-Ntoumani, 2011). No obstante, respecto a este último, en los diferentes ámbitos donde se ha estudiado, coinciden al apuntar en la misma línea, alertando de sus consecuencias negativas a nivel afectivo, cognitivo y comportamental para la persona (Castillo, González, Fabra, Mercé, y Balaguer, 2012).

Ya desde los primeros trabajos al respecto, situados en el ámbito familiar, el estilo controlador de los padres se relacionó con el desarrollo desadaptativo de los infantes (Soenens, Luyckx, Vanteenkiste, Duriez, y Goosens, 2008) señalando investigaciones posteriores, la tendencia hacia un autoconcepto negativo o comportamientos disruptivos en clase (Fuentes, 
Alarcón, y García, 2015). En otros contextos como el deportivo, los estudios también coinciden en señalar las consecuencias negativas o desadaptativas que genera en la persona el impacto del estilo de interacción controlador (Balaguer et al., 2012). En el ámbito educativo, el trabajo de Abós, Sevil, Sanz, Aibar y García-González (2016) indica que la falta de apoyo a la autonomía generada por el docente puede desencadenar en el estudiante conductas desinteresadas e indisciplinadas, probablemente generadas por la falta de iniciativa personal. Estos aspectos repercuten también en la disminución del procesamiento conceptual y creativo del estudiante (Grolnick y Ryan, 1987; Utman, 1997) así como desmotivación, menor utilización de estrategias meta-cognitivas (Soenens, Sierens, Vansteenkiste, Dochy, y Goossens, 2012), miedo o ansiedad (Hein, Koka, y Hagger, 2015). Por su parte, en el ámbito de la Educación Superior los estudios señalan además el declive del papel activo del estudiante respecto a etapas anteriores, en detrimento de la iniciativa o la creatividad (Martínez, 2004; López Aguado, 2004), por lo que el argumento anterior tomaría aún mayor sentido hacia el reclamo de estudios que profundicen en las necesidades este colectivo educativo.

Paralelamente a la figura de autoridad docente y a su estilo de interacción, el estudiante también puede utilizar dichos patrones de comportamiento durante su interacción con su grupo de iguales, por ejemplo, durante la gestión de una tarea académica. En este sentido, el estudiante que decide respetar y tener en cuenta la opinión de sus compañeros para la resolución de una tarea, escuchando las diferentes opiniones y permitiendo que cada uno se sienta responsable y satisfecho de su contribución estará practicando un estilo de apoyo a la autonomía con sus compañeros. Mientras, si la decisión es instruir a los demás compañeros mediante un enfoque más coercitivo imponiendo su propia forma de sentir, pensar y actuar para la gestión de una tarea, dicho estudiante responderá a un estilo controlador. Aunque es posible que la persona pueda recurrir a uno u otro estilo en momentos puntuales de su interacción atendiendo a las necesidades detectadas, incluso respondiendo a un estilo neutro, entendemos que de forma genérica existirá una tendencia personal que le situaría más cerca de uno u otro extremo (Pelletier, Fortier, Vallerand, y Briere, 2001; Tessier, Sarrazín, y Ntoumanis, 2008).

Desde la TAD, no encontramos hasta el momento instrumentos de medida enfocados a medir el estilo interaccional del estudiante, ya que los trabajos hasta el momento se han centrado en las figuras de autoridad. En este sentido, la Escala de Conductas Controladoras del Entrenador (CCBS) de Bartholomew, Ntoumanis, y Thøgersen-Ntoumani (2010) validada al contexto español por Castillo et al. (2014), mide la percepción que los deportistas tienen del estilo interpersonal controlador del entrenador. En el ámbito educativo, una de las escalas que contempla la tendencia del docente hacia el estilo controlador o de apoyo a la autonomía de sus estudiantes es la Problems in Schools Questionnaire (PIS) (Deci, Schwartz, Sheinman, y Ryan, 1981).

Por otro lado, aunque existe un gran número de trabajos actuales sobre los estilos de interacción en el contexto educativo a partir de la TAD (Abós et al., 2016) no hay evidencias respecto a la influencia de algunas variables personales como el sexo. Ciertamente, nos encontramos ante una variable cuyos resultados en la literatura, quizá fruto de limitaciones metodológicas, no nos ha permitido hasta el momento afirmar con seguridad el origen de las similitudes o diferencias entre chicos y chicas (Cano, 2000). Apoyados en la tripe vertiente de influencia que los patrones de interacción recogen del medio, la biología y las demandas propias de una situación concreta (Kolb, 1984) algunos trabajos justifican las semejanzas entre ambos 
sexos (Martínez, 1998), mientras que otros (Close y Shiel, 2009) recurren a la herencia de la construcción social de la persona para explicar las posibles diferencias encontradas. En este sentido, las escasas evidencias científicas se derivan del estudio de algunas variables relacionadas con los estilos de interacción que responden a los consecuentes comportamentales, cognitivos o emocionales con los que se han relacionado los estilos de apoyo a autonomía. Por ejemplo, en el estudio de López (2011) sobre los modos de abordar las situaciones de aprendizaje en universitarios, las mujeres se mostraron más reflexivas y los hombres más pragmáticos a la hora de gestionar sus aprendizajes. Otra aportación la hace Cano (2000) en su estudio sobre diferencias de género en estrategias y estilos de aprendizaje, donde señala la mayor actitud negativa de los chicos hacia el estudio. Sin embargo, como se indicó antes, con frecuencia la falta de consistencia empírica encontrada en la literatura ha llevado en ocasiones a apostar por la ausencia de diferencias respecto al sexo para la elección de la orientación o el patrón de uso de estrategias hacia el aprendizaje (Wilson, Smart, y Watson, 1996). Desde la TAD, las chicas vienen mostrando una mayor puntuación en la satisfacción de la necesidad de relación con los demás así como mayor motivación intrínseca (Severiens y Ten Dam, 1994). En el estudio de Grimes (1985) las chicas mostraron puntuaciones superiores a los chicos en actitud, motivación, administración del tiempo, ayudas al estudio y autocomprobación. Mientras en el trabajo de Gledhill y van Der Merwe's (1989) los chicos mostraron preferencia por las formas memorísticas que se orientaban al rendimiento y las chicas se decantaban por la orientación al significado.

Preocupados por la necesidad de seguir contribuyendo a la comprensión de estos aspectos, el desarrollo de un instrumento de medida del estilo interpersonal de control del estudiante supondría un primer paso para probar sus propiedades psicométricas en su versión española, pudiendo profundizar además su relación con la variable sexo del estudiante. En este sentido, a partir de los postulados teóricos presentados, los objetivos del estudio serán dos. En primer lugar, diseñar y validar un instrumento de medida para el estilo controlador en el estudiante. Y, en segundo lugar, determinar si existe una relación significativa entre el sexo de los estudiantes y el estilo de interacción elegido a la hora de gestionar un aprendizaje con sus compañeros. A partir de las indagaciones encontradas en la escasa evidencia empírica en este campo de trabajo, se espera que sean los chicos los que utilicen un estilo controlador en mayor medida que las chicas.

\section{Método}

\section{Participantes}

La muestra estuvo compuesta por 332 estudiantes universitarios (140 chicos y 192 chicas) de $2^{\circ}$ curso de grados pertenecientes al área de Ciencias de la Salud (Medicina, Farmacia, Psicología, Ciencias del Deporte, Podología y Terapia Ocupacional) en una Universidad española, con una edad media de 21.05 años $(D T=3.97)$.

\section{Medidas}

Estilo controlador. Se diseñó la Escala de Estilo Controlador del Estudiante (ECE) que finalmente quedó compuesta por ocho ítems que miden en un único factor el estilo controlador que perciben los estudiantes que transmiten en el entorno académico. Los ítems (e.g. "Presiono 
verbalmente a los demás para que se realice la actividad como yo he dicho") estaban precedidos por la sentencia previa "Yo como estudiante, cuando trabajo en pequeños grupos o en las clases de la universidad, respecto a mis compañeros y docentes...". Se midió a través de una escala tipo Likert que va desde 1 (Totalmente desacuerdo) a 5 (Totalmente de acuerdo).

\section{Procedimiento}

La construcción del cuestionario se realizó a partir de una revisión bibliográfica, donde se fueron construyendo los aspectos más importantes sobre los que debían girar los ítems. La elaboración de los ítems se centró en determinar si el estilo que los estudiantes percibían que utilizaban en el entorno académico era de tipo controlador. La redacción de los mismos surge de esa revisión para posteriormente someter la batería de ítems creada a una evaluación por parte de diez expertos en la temática, que puntuaron, en una escala Likert de cinco puntos sobre cada ítem de la escala, la suficiencia con el fin de ver si los ítems establecidos bastaban para medir su propósito, la claridad de lenguaje, la coherencia de estos y su relevancia teórica. Las conclusiones que se obtuvieron con esta evaluación fueron encaminadas a reescribir algunos ítems, en unos casos para mejorar su entendimiento y en otros para eliminar la dualidad aconsejándose separar. Seguidamente, se administró la batería completa a un reducido grupo de estudiantes $(n=32)$ para verificar su correcta comprensión. La impresión de los estudiantes fue que la escala no presentaba ningún problema de comprensión por lo que no se realizaron cambios. En la administración final a los estudiantes, se insistió en el anonimato de las respuestas para que contestaran con honestidad y sinceridad. El tiempo requerido para su cumplimentación fue de ocho minutos, aproximadamente.

\section{Análisis de datos}

Se calcularon los estadísticos descriptivos de todos los ítems objeto de estudio (medias y desviaciones típicas), se analizó la consistencia interna mediante el coeficiente de Alfa de Cronbach y las correlaciones bivariadas de todas las variables. La estructura de la escala se confirmó a través de un análisis factorial confirmatorio donde la validez del modelo de medición fue considerado a través de una serie de coeficientes fit, también llamados índices de bondad de ajuste: $\chi^{2}, \chi^{2} /$ d.f., RMSEA, RSMR y los índices incrementales (CFI, IFI y TLI). Asimismo, se comprobó a través de un ANOVA las posibles diferencias por sexo. Los datos fueron analizados mediante el paquete estadístico SPSS 21.0 y AMOS 21.0.

\section{Resultados}

\section{Análisis factorial confirmatorio de la $E C E$}

Se realizó un análisis factorial confirmatorio (CFA) basándonos en las nueve medidas observadas en el constructo estilo controlador, pero uno de los reactivos ("Cuando trabajo en pequeños grupos, doy la solución de cómo llegar a resolver la tarea") presentó un bajo peso de regresión estandarizado. Se utilizó el método de estimación de máxima verosimilitud junto con el procedimiento de bootstrapping, ya que el resultado del coeficiente multivariado de Mardia fue 26.79 , lo que indicaba falta de normalidad multivariada de los datos. Se realizó un nuevo CFA basándonos en ocho medidas observadas, obteniendo unos adecuados índices: $\chi^{2}(20, N=$ $332)=63.48, p<.000 ; \chi^{2} /$ d.f. $=3.17 ; \mathrm{CFI}=.93 ; \mathrm{IFI}=.90 ; \mathrm{TLI}=.93 ; \mathrm{RSMR}=.04 ; \mathrm{RMSEA}=$ 
.08. No obstante, se observó en los efectos indirectos que correlacionando los errores de los ítems uno ("Hablo continuamente y dejo muy poco margen para la aportación de los demás") y dos ("Cuando trabajo en pequeños grupos, doy explicaciones y respuestas sin permitir que los demás lo descubran por sí mismos") los índices mejoraban: $\chi^{2}(19, N=332)=40.47, p<.003$; $\chi^{2} /$ d.f. $=2.13 ;$ CFI $=.96 ;$ IFI $=.96 ;$ TLI $=.95 ;$ RSMR $=.03 ;$ RMSEA $=.05$ (Tabla 1$)$.

Tabla 1: Parámetros Estimados de AFC para la Escala Estilo Controlador

\begin{tabular}{|c|c|c|c|c|c|}
\hline & $M$ & $D T$ & Asimetría & Curtosis & PRE \\
\hline $\begin{array}{l}\text { Hablo continuamente y dejo muy poco margen } \\
\text { para la aportación de los demás }\end{array}$ & 2.04 & .94 & .78 & .23 & .49 \\
\hline $\begin{array}{l}\text { Cuando trabajo en pequeños grupos, doy } \\
\text { explicaciones y respuestas sin permitir que los } \\
\text { demás lo descubran por sí mismos }\end{array}$ & 2.11 & .97 & .62 & -.31 & .58 \\
\hline $\begin{array}{l}\text { En general, me aseguro de que los demás hagan } \\
\text { las cosas como yo quiero }\end{array}$ & 2.38 & 1.05 & .46 & -.45 & .64 \\
\hline $\begin{array}{l}\text { Decido fechas límite de entrega de las tareas } \\
\text { sin negociar el tiempo con los demás }\end{array}$ & 1.82 & .98 & 1.17 & .85 & .55 \\
\hline $\begin{array}{l}\text { Presto poca atención cuando los demás no se } \\
\text { esfuerzan en ver las cosas como yo digo }\end{array}$ & 2.17 & 1.01 & .58 & -.39 & .61 \\
\hline $\begin{array}{l}\text { Cuando algo no sale como quiero utilizo } \\
\text { expresiones como ¿Puedes hacerlo como te } \\
\text { expliqué? }\end{array}$ & 2.13 & 1.08 & .68 & -.34 & .55 \\
\hline $\begin{array}{l}\text { Cuando trabajo en pequeños grupos, animo } \\
\text { prometiendo recompensas a cambio de que lo } \\
\text { hagan como yo quiero }\end{array}$ & 1.64 & .98 & 1.55 & 1.64 & .57 \\
\hline $\begin{array}{l}\text { Presiono verbalmente a los demás para que se } \\
\text { realice la actividad como yo he dicho }\end{array}$ & 1.67 & .92 & 1.21 & .41 & .67 \\
\hline
\end{tabular}

Nota: PRE: Pesos de regresión;

\section{Análisis de consistencia interna}

La consistencia interna de la ECE se calculó utilizando el coeficiente alfa de Cronbach y se obtuvo un valor alfa de .79 para el estilo controlador.

\section{Análisis descriptivo y de correlación}

La dimensión estilo controlador presentó una media de $2.16(D T=.58)$ sobre un total de 5 puntos y mostró una correlación negativa con el sexo $(r=-.19 ; p>.001)$, siendo tabulados los chicos como "1" y las chicas como "2".

\section{Análisis diferencial}

Se realizó un ANOVA contemplando como variable dependiente la dimensión estilo controlador del estudiante y como variable independiente el sexo. Los resultados mostraron (C) Psy, Soc, \& Educ, 2017, Vol. 9(2) 
diferencias $(F(1,327)=12.62 ; p>.001)$, donde los chicos $(M=2.29 ; D T=.62)$ puntuaron más alto en estilo controlador que las chicas $(M=2.06 ; D T=.54)$.

\section{Discusión}

Se planteó como objetivo principal diseñar y validar la Escala de Estilo Controlador del Estudiante en población universitaria. Como objetivo secundario, se propuso relacionar el estilo de interacción social controlador del estudiante con el sexo hipotetizando que los chicos se identificarían en mayor medida que las chicas con el estilo controlador. Los resultados obtenidos confirman la hipótesis de partida.

Los resultados de este estudio señalan que la ECE en su versión de ocho ítems posee una buena consistencia interna, así como una adecuada validez de constructo, lo que informa que es posible contar con un instrumento adecuado para medir el estilo de interacción controlador que muestran los estudiantes de Educación Superior en su entorno académico. La escala presentó un ítem (ítem 9 "Cuando trabajo en pequeños grupos doy la solución de cómo llegar a resolver la tarea") cuya eliminación produjo un aumento de su consistencia interna. La razón pudo deberse a la falta de comprensión por el alumnado, debido, posiblemente a su carácter demasiado amplio. De esta forma, al eliminar este ítem, la escala aumentó la consistencia decidiendo mantener esta versión final de ocho ítems.

La principal razón de ser de este trabajo radica en el hecho de que en la actualidad, a pesar de las insistentes recomendaciones hacia el apoyo a la autonomía de la persona, son más frecuentes las prácticas de control (AssorKaplan, Kanat-Maymo, y Roth, 2005). Son varias las influencias que señalan los estudios para que la persona decida adoptar un estilo de interacción controlador, aunque dichos motivos solamente han sido referidos en la literatura hasta el momento a la figura de autoridad del docente (Hein, Koka, y Hagger, 2015). En este punto es importante delimitar el concepto de "comportamientos de control", ya que desde la TAD se conceptualiza como un estilo interpersonal que recibe influencias de diferentes fuentes externas (Hagger, 2014). Por ejemplo, se alude por un lado a agentes externos como las políticas educativas, las normas escolares, los padres y madres de los estudiantes, las expectativas sociales o las normas culturales. Por otro lado, existirían variables de naturaleza personal interna como las derivadas de las creencias del propio docente hacia su estilo de docencia eficaz o la coincidencia con las disposiciones de personalidad orientadas al control (Forstadt, 2007; Reeve, 2009). En definitiva, la mayoría de autores coinciden en señalar las presiones impuestas o autoimpuestas como la causa principal de la práctica del estilo controlador (Pelletier, Seguin-Levesque, y Legault, 2002).

Respecto a las diferencias por sexo en estudiantes, no existen trabajos que directamente hayan estudiado su relación con los estilos de apoyo a la autonomía o control, aunque en los que se han llevado a cabo respecto a la figura de autoridad del entrenador, no han encontrado diferencias por sexo (Trigueros, Aguilar, Fernández, Manzano, y Alcaraz, 2016). Sin embargo, los resultados de otros trabajos podrían ser relevantes para contrastar con nuestro hallazgo de que en estudiantes de Educación Superior, los chicos parecen manejar un estilo controlador en mayor frecuencia que las chicas. En este sentido, las investigaciones que han estudiado la variable sexo en relación a la TAD señalan que la necesidad de relación con los demás es más importante para las chicas (Ntoumanis, 2001), mostrando también una mayor puntuación en los 
tipos de motivación más intrínseca (Gené y Latinjak, 2014). Por lo que en el caso de los chicos, es posible que un estilo de interacción controlador les permita eludir esta interacción con sus compañeros para la gestión de las tareas en grupo, prefiriendo formas más coercitivas e impositivas de resolución de problemas hacia la consecución de recompensas de carácter más extrínseco (Gené y Latinjak, 2014). En este punto, el riesgo implícito a este escenario radica en qué a partir de los postulados de la TAD, alejarse del apoyo a la autonomía también implica un alejamiento de la satisfacción de las necesidades psicológicas básicas, y por ende, de la motivación autodeterminada (Vallerand y Losier, 1999).

Desde el ámbito educativo, este trabajo podría ser útil para el diseño y desarrollo de estrategias orientadas a minimizar el estilo controlador del estudiante a través del fomento de estrategias basadas en el apoyo a la autonomía, siendo especialmente recomendable en los primeros cursos de una titulación. Siguiendo los postulados de la TAD, el control percibido por los estudiantes podría mediante la frustración de las necesidades de competencia, autonomía y relación con los demás, generar sentimientos de resentimiento entre ellos, apatía incluso estrés, ansiedad o comportamientos de bullyng (Gené y Latinjak, 2014). Como indican algunos trabajos (Balaguer, Castillo, Duda, y Tomás, 2009; Pelletier, Fortier, Vallerand, y Briere, 2001) es necesario atender más allá de la adolescencia, el apoyo a la autonomía por los beneficios que conlleva. Además, cumplir con las exigencias de las competencias implica conocer al estudiante para poder adaptarse a su estilo de interacción a través de las herramientas docentes más ajustadas. Con ello, se fomentarían los beneficios generados por el estilo de apoyo a la autonomía produciendo una mayor adaptación a las demandas del ciclo educativo que se inicia.

Entre las limitaciones encontradas y respecto a las propiedades psicométricas de la Escala de Control del Estudiante (ECE), sería interesante examinar su invarianza utilizando diferentes edades, titulaciones, cursos e incluso teniendo en consideración su ajuste con el estilo de interacción docente. Especial relevancia cobra también en este punto el estudio de las variables individuales, como la autoeficacia (Brown, 2012; Huangfu, 2012) que podrían asociarse a cada uno de los estilos, de forma que puedan continuar el proceso de validez de este instrumento.

\section{Referencias}

Abós, A., Sevil, J., Sanz, M., Aibar, A., y García-González (2016). El soporte de autonomía en Educación Física como medio de prevención de la oposición desafiante del alumnado. RICYDE. Revista Internacional de Ciencias del Deporte, 43, 65-78.

Adie, J. W., Duda, J. L., y Ntoumanis, N. (2012). Perceived coach-autonomy support, basic need satisfaction and the well- and ill-being of elite youth soccer players: A longitudinal investigation. Psychology of Sport and Exercise, 13, 51-59.

Assor, A., Kaplan, H., Kanat-Maymon, Y., y Roth, G. (2005). Directly controlling teacher behaviors as predictors of por motivation and engagement in girls and boys: the role of anger and anxiety. Learning and Instruction, 15, 397e413.

Balaguer, I., Castillo, I., Duda, J. L., y Tomás, I. (2009). Análisis de las propiedades psicométricas de la versión española del Cuestionario de Clima en el Deporte. Revista de Psicología del Deporte, 18(1), 73-83. 
Balaguer, I., Gonzalez, L., Fabra, P., Castillo, I., Mercé, J., y Duda, J. L. (2012). Coaches' interpersonal style, basic psychological needs and the well- and ill-being of young soccer players: A longitudinal analysis. Journal of Sports Sciences, 30, 1-11.

Bartholomew, K. J., Ntoumanis, N., y Thøgersen-Ntoumani, C. (2010). Thecontrolling interpersonal style in a coachingcontext: Development and initialvalidation of a psychometric scale. Journal of Sport \& ExercisePsychology, 31, 193-216.

Bartholomew, K. J., Ntoumanis, N., Ryan, R. M., Bosch, J. A., y Thogersen-Ntoumani, C. (2011). Self-determination theory and diminished functioning: The role of interpersonal control and psychological needthwarting. Personality and Social Psychology Bulletin, 37(11), 1459-1473.

Bartholomew, K., Ntoumanis, N., y Thøgersen-Ntoumani, C. (2011). Self-determination theory and the darker side of Athletic experience: the role of interpersonal control and needthwarting. Sport and Exercise Psychology Review, 7, 23-27.

Black, A. E., y Deci, E. L. (2000). The effects of instructors' autonomy support and students' autonomous motivation on learning organi cchemistry: A self-determination theory perspective. Science Education, 84, 740-756.

Boggiano, A. K., Flink, C., Shields, A., Seelbach, A., y Barrett, M. (1993). Use of techniques promoting students' self- determination: Effects on students' analytic problem-solving skills. Motivation and Emotion, 17, 319-336. doi: 10.1007/BF00992323

Brown, C. G. (2012). A systematic review of the relationship between self-efficacy and burnout in teachers. Educational \& Child Psychology, 29(4), 47-63.

Cano, F. (2000). Diferencias de género en estrategias y estilos de aprendizaje. Psicothema, 12(3), 360-367.

Castillo, I., González, L., Fabra, P., Mercé, J., y Balaguer, I. (2012). Estilo interpersonal controlador del entrenador, frustración de las necesidades psicológicas básicas, y burnout en futbolistas infantiles. Cuadernos de Psicología del Deporte, 12(1), 143-146.

Castillo, I., Tomás, I., Ntoumanis, N., Bartholomew, K. J., Duda, J.L., y Balaguer, I. (2014). The Spanish version of the Controlling Coach Behaviors Scale in the Sport Context: Psychometric properties and invariance over time and competitive level. Psicothema, 26, 409-414.

Close, S., y Shiel, G. (2009). Gender and PISA Mathematics: Irish results in context. European Educational Research Journal, 8(1), 20-33.

Coatsworth, J. D., y Conroy, D. E. (2009). The effects of autonomy-supportive coaching, need satisfaction, and self-perceptions on initiative and identity in youths wimmers. Developmental Psychology, 45, 320-328.

Deci, E. L., y Ryan, R. M. (1985). Intrinsic motivation and self-determination in human behavior. Nueva York: Plenum Press.

Deci, E. L., y Ryan, R. M. (1987). The support of autonomy and the control of behavior. Journal of Personality and Social Psychology, 53, 1024-1037.

Deci, E. L., Schwartz, A., Sheinman, L., y Ryan, R. M. (1981). An instrument to assess adults' orientation stoward control versus autonomy in children: Reflections on intrinsic motivation and perceived competence. Journal of Educational Psychology, 73, 642-650. 
Forstadt, L. (2007). Swimming with thes harks: Basis of job satisfaction for teachers who educate at-risk, high-school students. Unpublished dissertation, University of Iowa, Iowa City.

García, J. L. (2006). Los Estilos de Aprendizaje y las Tecnologías de la Información y la Comunicación en la Formación del Profesorado. Tesis Doctoral. Dirigida por Catalina Alonso García. Madrid: Universidad Nacional de Educación a Distancia.

Gené, P. S., y Latinjak, A. T. (2014). Relación entre las necesidades básicas y la autodeterminación en deportistas de élite. Cuadernos de Psicología del Deporte, 14(3), 49-56.

Gledhill, R. F., y van Der Merwe, C. A. (1989). Gender as a factor in student learning: preliminary findings. Medical Education, 23, 201-204.

Grimes, S.K. (1995). Targeting academic programs to student diversity utilizing learning styles and learning-studys trategies. Journal of College Student Development, 36(5), 422-430.

Grolnick, W. S., y Ryan, R. M. (1987). Autonomy in children's learning: An experimental and individual difference investigation. Journal of Personality and Social Psychology, 52, 890-898.

Hagger, M. S. (2014). Avoiding the "dejà-variable" phenomenon: social psychology needs more guides to constructs. Frontiers in Psychology, 5, 52.

Hagger, M. S., y Chatzisarantis, N. L. D. (2007). Advances in self-determination theory research in sport and exercise. Psychology of Sport and Exercise, 8, 597-599. http://dx.doi.org/10.1016/j.psychsport.2007.06.003.

Hein, V., Koka, A., y Hagger, M. (2015). Relationships between perceived teachers' controlling behaviour, psychological needth warting, anger and bullying behaviour in high-school students. Journal of Adolescence, 42, 103-114.

Huangfu, W. (2012). Effects of EFL Teachers' Self-Efficacyon Motivational Teaching Behaviors. Asian Social Science, 8(15), 68-74.

Kolb, D. A. (1984). Experiential Learning. Experience as the Source of Learning and Development, Englewoodcliffs. New York: Prentice-Hall.

Ley Orgánica 1/1990, de 3 de octubre, de Ordenación General del Sistema Educativo (LOGSE), BOE, núm. 238, de 4 de octubre de 1990, (28927-28942).

Ley Orgánica 2/2006, de 3 de mayo, de Educación (LOE). BOE, núm. 106, de 4 de mayo de 2006, (17158-17207).

Ley Orgánica 8/2013, de 9 de diciembre, para la mejora de la calidad educativa (LOMCE). BOE, núm. 295, de 10 de diciembre de 2013, (97858-97921).

López-Aguado, M. (2004). Estilo de aprendizaje pragmático en estudiantes universitarios. Variables relacionadas y valoración diferencial del buen profesor en función del grado de preferencia. Actas del I Congreso Internacional de Estilos de Aprendizaje. Madrid: UNED.

Martínez, P. (2004). Investigación y análisis de los estilos de aprendizaje del profesorado y de sus alumnos del primer ciclo de Educación Secundaria Obligatoria (ESO) en el ámbito del CPR de Laredo. Cantabria. España. Actas del I Congreso Internacional de Estilos de Aprendizaje. Madrid: UNED.

Ntoumanis, N. (2001). A self-determination approach to the understanding of motivation in physical education. British Journal of Educational Psychology, 71, 225-242. 
Niemiec, C. P., Ryan, R. M., y Deci, E. L. (2009). The pathtaken: consequences of attaining intrinsic and extrinsic aspirations in post-college life. Journal of Research in Personality, 43, 291-306.

López-Aguado, M. (2004). Estilo de aprendizaje pragmático en estudiantes universitarios. Variables relacionadas y valoración diferencial del buen profesor en función del grado de preferencia. Actas del I Congreso Internacional de Estilos de Aprendizaje. Madrid: UNED.

López-Aguado, M. (2011). Estilos de aprendizaje. Diferencias por género, curso y titulación. Revista Estilos de Aprendizaje, 7(7), 109-134.

Pegalajar, M. C., y López, L. (2015). Competencias emocionales en el proceso de formación del docente de Educación Infantil. REICE. Revista Iberoamericana sobre Calidad, Eficacia y Cambio en Educación, 13(3), 95-106.

Pelletier, L. G., Fortier, M. S., Vallerand, R. J., y Briere, N. M. (2001). Associations among perceived autonomy support, forms of self-regulation, and persistence: A prospective study. Motivation and Emotion, 25, 279-306.

Pelletier, L. G., Seguin-Levesque, C., y Legault, L. (2002). Pressure from above and pressure from below as determinants of teachers' moti- vation and teaching behaviors. Journal of Educational Psychology, 94, 186-196.

Reeve, J. (2009). Why teachers adopt a controlling motivating style toward students and how they can become more autonomy suppor- tive. Educational Psychologist, 44(3), 159175. doi:10.1080/00461520 903028990

Severiens, S.E., y Ten Dam, G.T.(1994). Gender differences in learning styles: a narrative review and quantitative meta-analysis. Higher Education, 27, 487-501.

Soenens, B., Sierens, E., Vansteenkiste, M., Dochy, F., y Goossens, L. (2012). Psychologically controlling teaching: Examining outcomes, antecedents, and mediators. Journal of Educational Psychology, 104(1), 108-120.

Tessier, D., Sarrazin, P., y Ntoumanis, N. (2008). The effects of an experimental program metosupport students' autonomy on the overt behaviours of physical education teachers. European Journal of Psychology of Education, 23, 239-253.

Trigueros, R., Aguilar, J. M., Fernández, J. M., Manzano, A., y Alcaráz, M. (2016). Validación de la CCBS en actividad física en personas mayores de 55 años de edad. Actas del $I V$ Congreso Internacional de Deporte Inclusivo. Universidad de Almería.

Utman, C. H. (1997). Performance effects of motivational state: A metaanalysis. Personality and Social Psychology Review, 1, 170-182.

Vallerand, R. J. y Losier, G. F. (1999). An integrative analysis of intrinsic and extrinsic motivation in sport. Journal of Applied Sport Psychology, 11, 142-169.

Wilson, K. L., Smart, R. M., y Watson, R. J. (1996). Gender differences in approaches to learning in first year psychology students. British Journal of Educational Psychology, $66,59-71$. 